УДК 532.5: 536.24

\title{
ВЛИЯНИЕ ИОНОВ ПРИМЕСИ НА КОЛИЧЕСТВО ВОДОРОДНЫХ СВЯЗЕЙ
}

\author{
Авраменко А.А., чЛ.-кор. НАН Украины, Тыринов А.И., канд. техн. наук,
} Дмитренко Н.П., канд. техн. наук, Кравчук А.В.

Институт технической теплофизики НАН Украины, ул. Желябова, 2 а, Киев, 03680, Украина

Розглянуто вплив різної кількості іонів $\mathrm{Cl}^{-}, \mathrm{Na}^{+}$та $\mathrm{Na}^{+}+\mathrm{Cl}^{-}$на кількість водневих зв'язків в водному розчині. Наведено аналіз впливу часу на водневі зв'язки в водних розчинах 3 іонами домішок.
Рассмотрено влияние различного количества ионов $\mathrm{Cl}^{-}, \mathrm{Na}^{+}$, и $\mathrm{Na}^{+}+\mathrm{Cl}^{-}$на количество водородных связей в водном растворе. Проведен анализ влияния времени на водородные связи в водных растворах с ионами примеси.
The effect of various amounts of ions $\mathrm{Cl}^{-}, \mathrm{Na}^{+}$, and $\mathrm{Na}^{+}+\mathrm{Cl}^{-}$on the quantity of the hydrogen bonds in aqueous solution is investigated. The effect of time on the hydrogen bonds in aqueous solutions with impurity ions is analyzed.

Библ. 16, рис. 2.

Ключевые слова: компьютерное моделирование, молекулярная динамика, водородная связь, ионы примесей, молекулы.

$a$-ускорение;

$F$ - сила;

$m$ - масса атома;

$n$ - количество водородных связей;

$t$ - время;

$U$-потенциальная энергия;

$x$ - координата;

$v$ - скорость движения;

\section{Индексы:}

В - химическая связь;

\section{Введение}

Анализ динамики образования и разрыва водородных связей, является интересной задачей при молекулярном исследовании различный химических растворов. Водородная связь является видом химического свойства атома водорода, которое позволяет соединить (объединить) его и другой электороотрицательный атом. Указанная особенность проявляется в виде межмолекулярных и внутримолекулярных взаимодействий. Наличие водородных связей в веществе существенно влияют на его физические (температура кипения, вязкость, летучесть) и химические (кислотность) свойства. Образование водородных связей с молекулами растворителя увеличивает растворимость вещества [1].

В настоящее время существуют различные способы исследования образования и разрыва водородных связей. Одними из наиболее распро- el - электростатика;

$h b$ - водородная связь;

$L J$ - взаимодействие Ван-дер-Ваальса;

$n$ - количество водородных связей;

$v$ - валентные углы;

$\kappa$ - количество атомов примеси;

$\varphi-$ торсионные углы;

$\omega-$ плоская группа.

страненных являются методы спектрографии [2, 3], а также моделирование с помощью различных пакетов молекулярной динамики (МД) [4 - 8]. Используя МД, авторы работы [9] исследовали различные свойства водородной связи и время их жизни. Рассматривались как случаи с чистым водным раствором, так и при наличии в указанном растворе ионов примеси при различных температурных условиях.

В работе [10] используя экспериментальные (спектральные) и компьютерные (МД) методы рассматривалась динамика поведения водородных связей в водном растворе $\mathrm{Na}^{+}+\mathrm{Cl}^{-}$при температуре $25 \ldots 500{ }^{\circ} \mathrm{C}$ и давлении 1000 бар.

В настоящем исследовании применялся метод молекулярной динамики для выявления влияния на количество водородных связей различного количества примесей ионов $\mathrm{Cl}^{-}, \mathrm{Na}^{+}$и $\mathrm{Na}^{+}+\mathrm{Cl}^{-}$. 
Метод МД был выбран из-за того, что он позволяет получить реальную информацию о строении и взаимодействии молекул того или иного вещества, а также дает возможность понять, или спрогнозировать протекание тех или иных физических и химических процессов внутри исследуемого микрообъекта. Также методы компьютерного моделирования (к которым относят МД) дают возможность связать между собой теоретические и экспериментальные наработки, особенно в тех случаях, когда аналитически сложно исследовать поведение молекул.

Метод МД стали интенсивно применять для исследования молекулярной структуры воды с конца 60-х годов прошлого столетия. Впервые метод МД предложен был в $[11,12]$. Методами МД старались адекватно описать радиальные корреляционные функции распределения плотности, получаемые с помощью рентгеновской и нейтронной дифракции. Современное развитие компьютерной техники значительно расширило области использования МД. С помощью МД можно проанализировать химические и физические свойства различных веществ и материалов на наноуровне, а также особенности динамики их взаимодействия с другими средами. Указанный метод позволяет вычислить траектории движения отдельных атомов. МД дает возможность рассматривать процессы во временных масштабах в несколько наносекунд, где электростатические взаимодействия более существенны по сравнению с квантовыми эффектами.

\section{Математическая модель}

В настоящее время общепринятой является следующая схема метода МД: рассматривается система с определенным количеством частиц и с заданным потенциалом взаимодействия между ними. В основу метода входит численное решение классических уравнений движения частиц (1) в некотором выделенном объеме среды. Для каждого атома записывается уравнение движения в виде второго закона Ньютона:

$m_{i} \frac{d^{2} x_{i}}{d t^{2}}=F_{i}(x), i=1,2, \ldots, N$.

Предполагается, что все силы носят консервативный характер. Поэтому имеем
$F_{i}(x)=-\frac{\partial U(x)}{\partial x_{i}}$,

где $U(x)$ - потенциальная энергия, зависящая от взаимного расположения всех атомов.

Мгновенная температура в МД моделировании определяется как средняя кинетическая энергия, приходящаяся на одну степень свободы расчетной системы:

$T(t)=\frac{1}{3 N k_{B}} \sum_{i=1}^{N} m_{i} v_{i}^{2}$,

где $k_{B}$ - константа Больцмана. Температура среды определяется усреднением ее мгновенных значений $T(t)$ по некоторому интервалу времени.

Потенциальная энергия, которая входит в уравнение (1), описывается выражением:

$$
U(x)=U_{B}+U_{v}+U_{\varphi}+U_{\omega}+U_{L J}+U_{e l}+U_{h b},
$$

При использовании математической модели (1) - (4) для моделирования процессов молекулярной динамики задаются периодические граничные условия, т.е. молекулы и атомы, покидающие расчетную область, вносятся в нее через противоположную границу.

Обычно при МД расчете используется алгоритм Верле [13], когда положение точки может быть вычислено без знания скорости. Данный алгоритм является компромиссом между точностью процедуры расчета и скоростью ее реализации.

Последовательность расчета имеет вид:

$$
\begin{array}{ll}
a_{i}(t)=\frac{F_{i}(x, t)}{m_{i}}, & i=1,2, \ldots, N . \\
v_{i}(t+\Delta t)=v_{i}(\Delta t)+a_{i}(t) \Delta t, & i=1,2, \ldots, N . \\
x_{i}(t+\Delta t)=x_{i}(t)+v_{i}(t) \Delta t+a_{i}(t) \frac{\Delta t^{2}}{2}, & i=1,2, \ldots, N .
\end{array}
$$

Для устранения эффекта нереального ускорения молекул воды, что обусловлено видом уравнений (1), при моделировании методом МД расчет проводят, учитывая условие постоянства температуры. С этой целью применяют различные приемы для поддержания постоянной температуры, используя различные способы задания термостата $[14,15]$. Одним из распространенных термостатов, используемых в молекулярной 
динамике, является термостат Ланжевена [16].

При учете термостатирования уравнение движения будет иметь вид:

$m \frac{d^{2} x}{d t^{2}}=-\nabla U-\gamma m v_{i}+F^{+}$,

Уравнение (8), в отличие от классических уравнений МД, включает две дополнительные силы: диссипативную силу $\gamma m v_{i}$ и стохастическую силу $F^{+}(\gamma-$ коэффициент сопротивления). Эти две силы взаимосвязаны флуктационо-диссипативной теоремой [14]. Корреляция случайной силы определяется выражением:

$$
\left\langle F_{i}^{+}\left(t_{1}\right) F_{j}^{+}\left(t_{2}\right)\right\rangle=2 \gamma k_{B} T \delta_{i j} \delta\left(t_{1}-t_{2}\right) \text {, }
$$

где $\delta_{i j}$ - символ Кронекера, $\delta$ - дельта-функция. Уравнение (9) представляет собой стохастическое уравнение Ланжевена. Для его численного решения используется метод Монте-Карло, суть

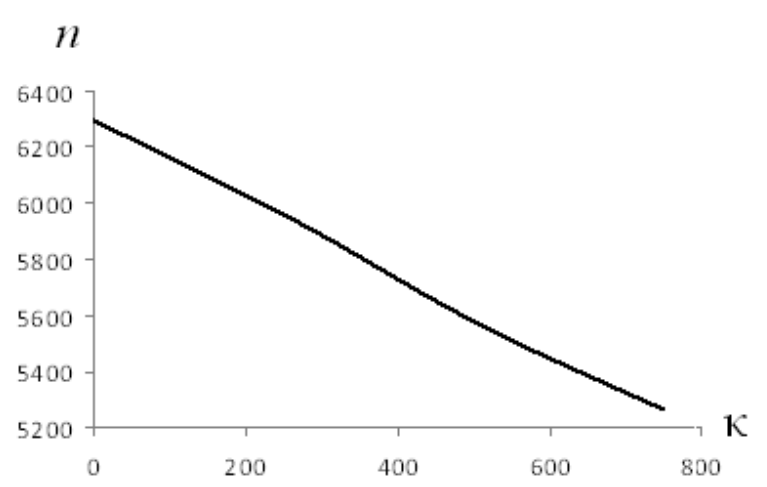

a) которого состоит в интегрировании по случайной выборке атомов, а не по всей системе.

\section{Физическая постановка задачи}

На основе изложенной методики было проведено компьютерное моделирование влияния различного количества примесей в воде, а именно, ионов $\mathrm{Cl}^{-}, \mathrm{Na}^{+}$и $\mathrm{Na}^{+}+\mathrm{Cl}^{-}$на образование или разрыв водородных связей. Рассматриваемый водный раствор (чистый) состоял из 10 тыс. молекул, в который вводилось разное количество ионов примеси. Исследовались четыре варианта конфигураций: первый - чистая среда (только молекулы воды), второй - в водные растворы вводятся 250 ионов $\mathrm{Cl}^{-}, \mathrm{Na}^{+}$и $\mathrm{Na}^{+}+\mathrm{Cl}^{-}$, третья - в воду вводятся 500 ионов $\mathrm{Cl}^{-}, \mathrm{Na}^{+}$и $\mathrm{Na}^{+}+\mathrm{Cl}^{-}$, четвертая - в воду вводятся 750 ионов $\mathrm{Cl}^{-}, \mathrm{Na}^{+}$и $\mathrm{Na}^{+}+\mathrm{Cl}^{-}$. Системы рассматривалась при температуре $298 \mathrm{~K}$ с периодическими граничными условиями.

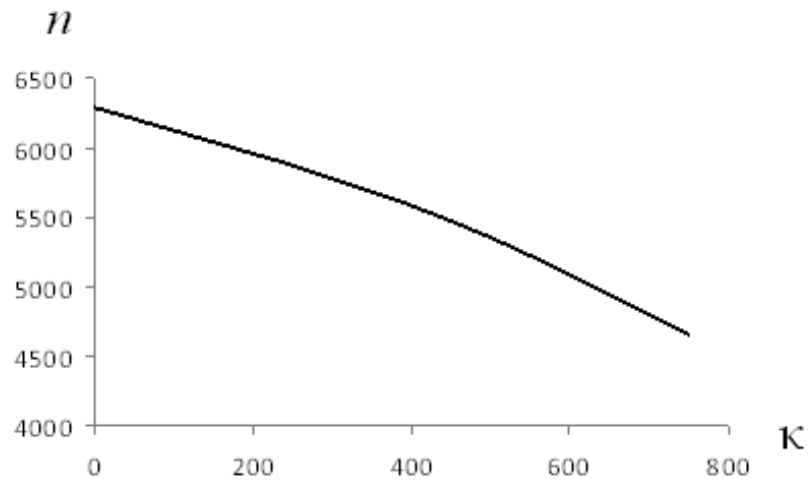

б)

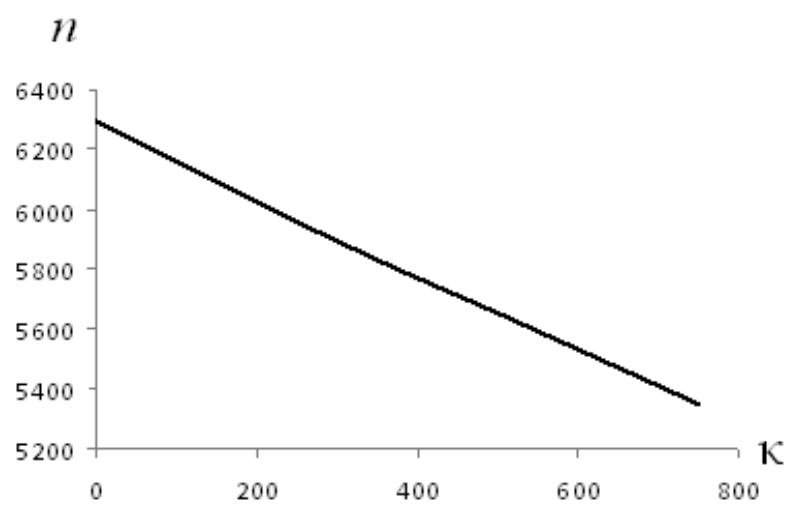

b)

Рис. 1. Зависимость количества водородных связей от концентрации ионов примесей: a) $-\mathrm{Cl}$; б) $-\mathrm{Na}^{+}$; и в $)-\mathrm{Na}^{+}+\mathrm{Cl}$. 
На рис. 1 представлены результаты исследования зависимости количества водородных связей от различной концентрации ионов примесей $\mathrm{Cl}^{-}(\mathrm{a}), \mathrm{Na}^{+}$(б) и $\mathrm{Na}^{+}+\mathrm{Cl}^{-}$(в). Из рисунков видно, что ионы примесей оказывают существенное влияние на количество водородных связей, а именно, их увеличение способствует уменьшению количества водородных связей. Ионы примесей не образуют водородные связи. Такой эффект скорее всего связан с диполь-дипольным взаимодействием. Водородная связь имеет электростатическую компоненту. В отличие от нее диполь-дипольное взаимодействие состоит в ковалентной составляющей. Если рассматривать случай, где примесью являются ионы хлора, то там нет или очень мало орбитального смешивания между наивысшей молекулярной орбиталью $\mathrm{Cl}^{-}$и наинизшей орбиталью Н. А без орбитального смешивания водородные связи не образуются. Существует только диполь-дипольное взаимодействие.

Уменьшение количества водородных связей в рассмотренных растворах с увеличением концентрации примесей объясняется еще и тем, что введенные ионы имеют существенные размеры, и их добавление в чистый раствор способствует заполнению пространства, необходимого для образования указанных связей.

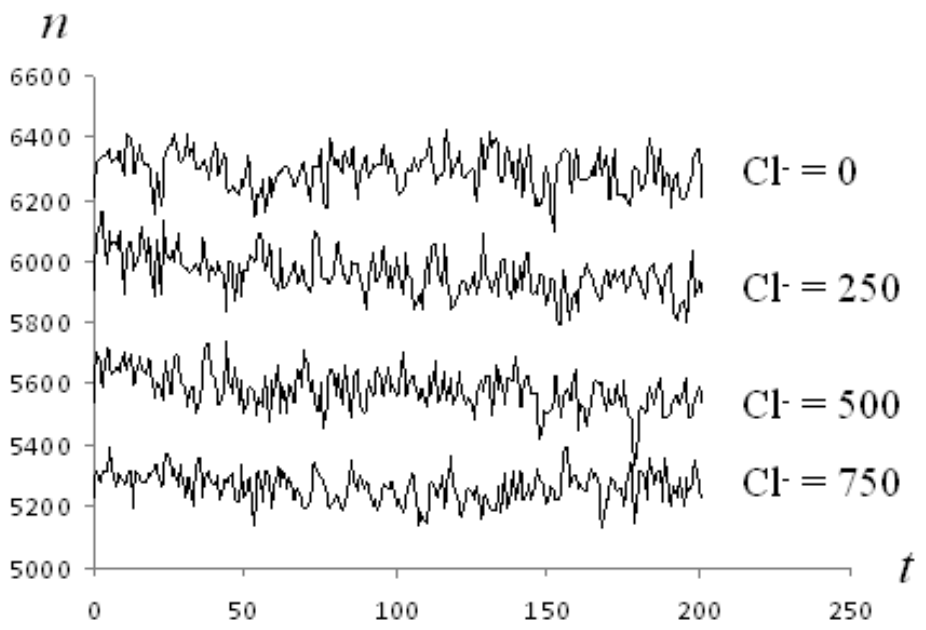

Рис. 2. Зависимость количества водородных связей от концентрации ионов $\mathrm{Cl}$.

Также рассматривались случаи влияния времени на количество водородных связей в водных растворах с ионами $\mathrm{Cl}^{-}, \mathrm{Na}^{+}$и $\mathrm{Na}^{+}+\mathrm{Cl}^{-}$. Как видно из рис. 2 , временной фактор не оказывал существенного влияния на образование или разрыв водородных связей в водном растворе с различной концентрацией примесей $\mathrm{Cl}^{-}$. В статье приведены результаты моделирования только для водного раствора с ионами $\mathrm{Cl}^{-}$, так как для других рстворов характер результатов идентичен.

\section{Выводы}

В настоящей работе приведены результаты компьютерного исследования влияния ионов примесей на количество водородных связей в водном растворе методом МД. Установлено, что увеличение концентрации ионов примесей при- водит к уменьшению количества водородных связей. При этом временной фактор не оказывает какого-либо существенного влияния. Из результатов также видно, что наибольшее влияние на уменьшение водородных связей в водном растворе оказывают ионы $\mathrm{Na}^{+}+\mathrm{Cl}^{-}$.

\section{ЛИТЕРАТУРА}

1. Ахметов Н.С. Общая неорганическая химия: Учебник для вузов / Н.С. Ахметов. - М.: Высш. Школа, 1981. - 679 с.

2. Казииина Л.А., Куплетская Н.Б. Применение Уф, ИК, и ЯМР - спектрографии в органической химии / Л.А. Казицина, Н.Б. Куплетская. - М.: изд. Высш. Школа, 1971, - 264 с.

3. Отто М. Современные методы аналитической химии / М. Отто. - М.: Техносфера, 2008 
$-544 \mathrm{c}$.

4. Quirke N. Adsorption and transport at the nanoscalle / N. Quirke. - London -New York: Boca Raton, 2006. - 189 p.

5. MacElroy J.M.D. Nonequilibrium molecular dynamics simulation of diffusion and flow in thin microporous membranes // Journal of chemical physics. - 1994. - V. 101, N 6. - P. 5274-5280.

6. Frenkel D., Smit B. Understanding molecular simulation / D.Frenkel, B.Smit. - London: Academic Press, 1996. - 443 p.

7. Rapaport D.C. The art of molecular dynamics simulation / D.C. Rapaport. - London: Cambridge University Press, $-1996 .-400$ p.

8. Товбин Ю.К. Метод молекулярной динамики в физической химии / Ю.К. Товбин - М.: Наука, - 1996. - 334 с.

9. Chandra $A$. Structure and dynamics of hydrogen bonds in liquid water and aqueous solutions // Proc. Indian Nat. Sci. Akad. - 2003. - N 1. - pp. 49-59.

10. Bondarenko G.V., Gorbaty Yu. E., Okhulkov $A . V$. Structure and hydrogen bonding in liquid and supercritical aqueous $\mathrm{NaCl}$ solutions at pressure
1000 bar and temperature up to $500{ }^{\circ} \mathrm{C}$ : a comprehensive experimental ana computational study // J. Chem. Phys. - 2006. - V. 110. - pp. 40424052.

11. Alder, B. J., and Wainwright, T. E. Phase transition for hard sphere system // J. Chem. Phys. 1957. - V. 2. - pp. 1208-1209.

12. Alder, B. J., and Wainwright, T. E. Studies in molecular dynamics. I. General method // J. Chem. Phys. - 1959. - V. 31. - pp.459-466

13. Verlet L. Computer experiments on classical fluids. Thermodynamic properties of Lenerd-Jons molecules // Phys. Rev. -1967. - V. 159. - P. 98-103.

14. Allen M.P., Tildesley D.J. Computer Simulation of Liquids / M.P Allen, D.J. Tildesley Oxford: Clarendon Press, - 1988. - 387 p.

15. Frenkel D., Smit B. Understanding Molecular Simulation: From Algorithms to Applications / D. Frenkel, B. Smit - San Diego: Academic Press, 2002. $-638 \mathrm{p}$.

16. Heo S.J., Sinnott S.B., Brenner D.N., Harrison J.A. Nanotribology and nanomechanics / S.J. Heo, S.B. Sinnott, D.N. Brenner, J.A. Harrison Berlin: Springer, $-2005 .-621 \mathrm{p}$. 


\section{THE INFLUENCE OF IMPURITIES ON THE NUMBER OF HYDROGEN BONDS}

\section{Avramenko A.A., Tyrinon A.I., Dmitrenko N.P., Kravchuk A.V.}

Institute of Engineering Thermophysics of the National Academy of Sciences of Ukraine, vul. Zhelyabova, 2a, Kyiv, 03680 Ukraine

The article the influence of different amounts of impurity ions, namely, $\mathrm{Cl}^{-}, \mathrm{Na}^{+}, \mathrm{NaCl}$ on hydrogen bonds in aqueous solution is investigated. The study was conducted using the method of molecular dynamics. It is found that increasing the concentration of the impurity ions reduces the amount of hydrogen bonding. At this time factor does not appear to have any significant effect. From the results also clear that the greatest impact on the reduction of the hydrogen bonds in aqueous solution having ions $\mathrm{NaCl}$.

References 16, figures 2.

Key words: computer simulation, molecular dynamics, hydrogen bond, impurity ions, molecules.

References 16. figures 2.

1. Akhmetov N.S. General Inorganic Chemistry: Textbook for Universities / N.S. Akhmetov. - M.: Higher School, 1981. - 679 p.

2. Kazitsina L.A., Kupletskaya N.B. Application of UV, IR, and NMR - Spectrograph in organic chemistry / L.A. Kazitsina, NB Kupletskaya. - M .: Ed. Executive. School, $1971-264$ p.

3. Otto $M$. Modern methods of analytical chemistry / M. Otto. - M .: Technosphere, 2008 $544 \mathrm{p}$.

4. Quirke N. Adsorption and transport at the nanoscalle / N. Quirke. - London -New York: Boca Raton, 2006. - 189 p.

5. MacElroy J.M.D. Nonequilibrium molecular dynamics simulation of diffusion and flow in thin microporous membranes // Journal of chemical physics. - 1994. - V. 101, N 6. - P. 5274-5280.

6. Frenkel D., Smit B. Understanding molecular simulation / D.Frenkel, B.Smit. - London: Academic Press, 1996. - 443 p.

7. Rapaport D.C. The art of molecular dynamics simulation / D.C. Rapaport. - London: Cambridge University Press, $-1996 .-400$ p.

8. Tovbin J.K. The method of molecular dynamics in physical chemistry / J.K. Tovbin - M.: Nauka, 1996. - $334 \mathrm{p}$.

9. Chandra $A$. Structure and dynamics of hydrogen bonds in liquid water and aqueous solutions // Proc. Indian Nat. Sci. Akad. - 2003. - N 1. - pp. 49-59.

10. Bondarenko G.V., Gorbaty Yu. E., Okhulkov $A$. $V$. Structure and hydrogen bonding in liquid and supercritical aqueous $\mathrm{NaCl}$ solutions at pressure 1000 bar and temperature up to $500{ }^{\circ} \mathrm{C}$ : a comprehensive experimental ana computational study // J. Chem. Phys. - 2006. - V. 110. - pp. 4042-4052.

11. Alder, B. J., and Wainwright, T. E. Phase transition for hard sphere system // J. Chem. Phys. 1957. - V. 2. - pp. 1208-1209.

12. Alder, B. J., and Wainwright, T. E. Studies in molecular dynamics. I. General method // J. Chem. Phys. - 1959. - V. 31. - pp.459-466

13. Verlet L. Computer experiments on classical fluids. Thermodynamic properties of Lenerd-Jons molecules// Phys. Rev. -1967. - V. 159. - P. 98-103.

14. Allen M.P., Tildesley D.J. Computer Simulation of Liquids / M.P Allen, D.J. Tildesley Oxford: Clarendon Press, - 1988. - 387 p.

15. Frenkel D., Smit B. Understanding Molecular Simulation: From Algorithms to Applications / D. Frenkel, B. Smit - San Diego: Academic Press, 2002. -638 p.

16. Heo S.J., Sinnott S.B., Brenner D.N., Harrison J.A. Nanotribology and nanomechanics / S.J. Heo, S.B. Sinnott, D.N. Brenner, J.A. Harrison - Berlin: Springer, -2005 . $-621 \mathrm{p}$. 